\title{
Coeficientes médios da equação de Angström-Prescott, radiação solar e evapotranspiração de referência em Brasília
}

\author{
Karla Daniele e Silva Dornelas ${ }^{(1)}$, Cícero Lopes da Silva( ${ }^{(2)}$ e Carlos Alberto da Silva Oliveira ${ }^{(2)}$
}

(1)QNL 19, conjunto J, casa 17, CEP 72152-010 Taguatinga, DF. E-mail: karladornelas@gmail.com (2)Universidade de Brasília, Fac. de Agronomia e Medicina Veterinária, Caixa Postal 04508, CEP 70919-970 Brasília, DF. E-mail: cicero@unb.br, dasilvao@unb.br

\begin{abstract}
Resumo - O objetivo deste trabalho foi estimar os coeficientes médios $a$ e $b$ da equação de Angström-Prescott, em Brasília, e seus efeitos sobre o cálculo da radiação solar global $\left(\mathrm{R}_{\mathrm{s}}\right)$ e da evapotranspiração de referência (ETo). Foram utilizados dados da estação meteorológica da Embrapa Hortaliças, do período de 1978 a 2003. Os coeficientes médios foram determinados por análise de regressão linear, no período todo e em períodos mensais e anuais. Utilizando-se o coeficiente calculado e os propostos pela FAO $(0,25$ e 0,50$)$ estimaram-se $R_{s}$ e ETo, e avaliaram-se os seus desempenhos. Os coeficientes médios mensais $a$ variaram de 0,241 a 0,345, e $b$ de 0,430 a 0,515. Os coeficientes médios anuais $a$ e $b$ foram 0,278 e 0,498, e os do período geral foram 0,282 e 0,490, respectivamente. A performance entre esses coeficientes na estimativa da radiação não diferiu. No mês de julho, foram verificados as piores estimativas e os piores desempenhos. Com as estimativas de $\mathrm{R}_{\mathrm{s}}$ e os coeficientes médios calculados, as estimativas de ETo não diferiram dos observados, tendo-se constatado um ótimo desempenho, independentemente do coeficiente utilizado. Dependendo do tipo de aplicação final dos dados, recomenda-se utilizar, por sua simplicidade, os coeficientes médios obtidos para o período geral.
\end{abstract}

Termos para indexação: balanço de energia, duração de brilho solar, Penman-Monteith-FAO.

\section{Angström-Prescott equation mean coefficients, solar radiation and reference crop evapotranspiration in Brasília}

\begin{abstract}
The objective of this work was to estimate Angström-Prescott equation mean coefficients in Brasília, $\mathrm{DF}$, Brazil, and their effects on the calculations of global radiation $\left(\mathrm{R}_{\mathrm{s}}\right)$ and reference crop evapotranspiration (ETo). It was used meteorological data from Embrapa Hortaliças weather station, from 1978 to 2003 . The mean coefficients were determined by linear regression analysis considering monthly, annual and total periods. Using the calculated coefficients for different periods and those provided by FAO (0.25 and 0.50), $\mathrm{R}_{\mathrm{s}}$ and ETo were estimated and their performances were evaluated. Monthly mean coefficient $a$ varied from 0.241 to 0.345 , and $b$ from 0.430 to 0.515 . Annual mean coefficients $a$ and $b$ were 0.278 and 0.498 , and total means were 0.282 and 0.490 , respectively. These coefficients did not differ from each other on radiation estimates. The month of July provided the worse estimates and performance. When using calculated $\mathrm{R}_{\mathrm{s}}$ estimates with monthly, annual, total and FAO coefficients, estimated values of ETo did not differ from observed ones, providing excellent performance, despite the mean coefficient used. Depending on the final application, it is suggested the use of total mean coefficients due to their easiness to estimate the shortwave radiation.
\end{abstract}

Index terms: energy balance, actual duration of sunshine, FAO-Penman-Monteith.

\section{Introdução}

Nos últimos anos, tem-se observado má distribuição das chuvas, em algumas regiões do país, o que provoca sérios problemas para a agricultura e para o abastecimento urbano de água. Tornou-se, então, necessário desenvolver métodos de uso racional de água para diminuir o desperdício, principalmente em sistemas de irrigação.
Um dos principais parâmetros climatológicos que afeta a evaporação da água do solo e a transpiração das plantas é a radiação solar, fator condicionante para a temperatura do ar e do solo (Pereira et al., 2002). Em relação às práticas de manejo da água na agricultura, o saldo de radiação solar é um dos principais elementos atuantes na perda de água pelos vegetais, na decomposição da matéria orgânica e nos processos metabólicos e biológicos (Ometto et al., 1973; Critchfield, 1974). 
De acordo com Fontana \& Oliveira (1996), a radiação solar global $\left(\mathrm{R}_{\mathrm{s}}\right)$ é um elemento meteorológico importante para o cálculo da evapotranspiração, realização de zoneamentos agroclimáticos, estabelecimento e utilização de modelos de crescimento e produção na agricultura. O grande problema para medir o saldo de radiação solar, nas estações meteorológicas, é o alto custo dos instrumentos a serem instalados e sua manutenção, que requer calibrações contínuas.

A radiação solar é medida por instrumentos como os radiômetros, actinógrafos bimetálicos e piranômetros, que também medem a radiação difusa, sendo este último o mais utilizado no Brasil. Quando a $\mathrm{R}_{\mathrm{s}}$ não está disponível no local, ela pode ser estimada com maior precisão, sendo necessário determinar os coeficientes de regressão $a$ e $b$ da equação de Angström-Prescott para os respectivos locais. Esses coeficientes dependem, principalmente, das características físicas da camada atmosférica a ser atravessada pela radiação solar e são influenciados pela latitude e altitude do local e pela época do ano. Quanto maior a latitude, maior a amplitude da radiação solar extraterrestre, entre o verão e o inverno (Pereira et al., 2002).

Vianello \& Alves (1991) apresentam valores médios anuais dos coeficientes da equação de AngströmPrescott, para diversas localidades do Brasil, por exemplo: para Salvador, valores de 0,278 e 0,498, e para Viçosa, MG, valores de 0,290 e 0,390, respectivamente, para $a$ e $b$. Essas localidades se situam em latitudes de $12^{\circ} 58^{\prime} \mathrm{S}$ e $20^{\circ} 45^{\prime} \mathrm{S}$, com altitudes de $8 \mathrm{~m}$ e $650 \mathrm{~m}$, respectivamente. No entanto, não foram encontradas, na literatura, informações sobre os coeficientes de Angström-Prescott para Brasília.

Uma revisão nos métodos de estimativa da evapotranspiração de referência (ETo) foi realizada por especialistas em 1990, que concluíram que para diversas condições de clima os resultados de ETo, calculados pela equação de Penman-Monteith, apresentavam resultados mais próximos daqueles oriundos de lisímetros de pesagem (Allen et al., 1998), o que sugeria que o método de Penman-Monteith se tornaria padrão para a estimativa da ETo.

Este trabalho teve como objetivo estimar os coeficientes médios $a$ e $b$ da equação de Angström-Prescott $\mathrm{e}$ seus efeitos sobre o cálculo da radiação solar global de ondas curtas e da evapotranspiração de referência, em Brasília.

\section{Material e Métodos}

Os dados meteorológicos foram coletados na Embrapa Hortaliças, situada em Brasília, a 1556'S, 48²'W e altitude de 997 m. O clima da região, segundo Köppen, é do tipo Cwa (mesotérmico, com inverno seco e verão quente). A precipitação média anual é de $1.360 \mathrm{~mm}$, com temperaturas médias anuais: máxima de $26,7^{\circ} \mathrm{C}$ e mínima de $16,1^{\circ} \mathrm{C}$.

A $\mathrm{R}_{\mathrm{S}}$ foi medida no solo, de 1991 a 1997, por uma estação automática, e de 1978 a 1990, e 1998 a 2003, por piranômetro de fotodiodo de silício, num total de 25 anos. A insolação (n), medida por um heliógrafo, a temperatura média do ar (T), a umidade relativa média do ar (UR) e a velocidade do vento, tomado a $2 \mathrm{~m}$ de altura $\left(\mathrm{u}_{2}\right)$, também fizeram parte das variáveis utilizadas, medidas em estação convencional e automática, conforme os períodos mencionados anteriormente.

Para a determinação dos coeficientes $a$ e $b$ da equação de Angström-Prescott, foram utilizados dados meteorológicos diários. Os coeficientes médios foram determinados em três períodos de tempo: mensal, anual e geral (que compreendeu toda duração da série, sem divisão por ano ou meses).

Os coeficientes médios gerais basearam-se em uma análise de regressão linear, com todos os dados diários de $n$ e $R_{s}$ medidos no período. Os coeficientes médios anuais foram analisados por meio de regressão linear, com os dados de cada ano. A média dos 25 coeficientes anuais $a$ e $b$ forneceu a média anual. Para cada mês foram geradas 25 equações, tendo-se calculado, ao final, um valor médio mensal de cada coeficiente, e tendo-se gerado, ao todo, 300 equações de regressão, para se determinar os 12 coeficientes mensais.

A equação de Angström-Prescott correlaciona a radiação $\mathrm{R}_{\mathrm{S}}$ com a razão de insolação $(\mathrm{n} / \mathrm{N})$, por meio da expressão:

$\mathrm{R}_{\mathrm{s}} / \mathrm{R}_{\mathrm{a}}=\mathrm{a}+\mathrm{b}(\mathrm{n} / \mathrm{N})$,

em que $\mathrm{R}_{\mathrm{s}}$ é a radiação solar global $\left(\mathrm{MJ} \mathrm{m}^{-2} \mathrm{~d}^{-1}\right)$; $\mathrm{n}$ representa as horas de insolação real $\left(\mathrm{h} \mathrm{d}^{-1}\right) ; \mathrm{N}$ é a duração máxima possível do brilho solar ( $\left.\mathrm{h} \mathrm{d}^{-1}\right) ; \mathrm{R}_{\mathrm{a}}$ é a radiação extraterrestre $\left(\mathrm{MJ} \mathrm{m}^{-2} \mathrm{~d}^{-1}\right)$; $a$ é o coeficiente que expressa a fração da radiação extraterrestre, que atinge a terra em dias totalmente nublados, correspondente à fração difusa; $b$ é o coeficiente complementar que expressa o total de radiação solar global. 
O saldo de radiação foi calculado com os dados de $\mathrm{R}_{\mathrm{s}}$ observados e estimados pelos coeficientes $a$ e $b$, obtidos e estimados com os valores médios anuais 0,25 e 0,50, respectivamente, sugeridos pela FAO (Allen et al., 1998).

Utilizou-se a metodologia da FAO (Allen et al., 1998), para se calcular a evapotranspiração de referência pelo método de Penman-Monteith-FAO:

ETo $=\left\{0,408 \Delta\left(\mathrm{R}_{\mathrm{n}}-\mathrm{G}\right)+\gamma[900 /(\mathrm{T}+273)] \mathrm{u}_{2}\left(\mathrm{e}_{\mathrm{s}}-\mathrm{e}_{\mathrm{a}}\right)\right\} /$ $\left[\Delta+\gamma\left(1+0,34 \mathrm{u}_{2}\right)\right]$,

em que ETo é a evapotranspiração de referência $\left(m m d^{-1}\right) ; R_{n}$ é a radiação líquida ou saldo de radiação $\left(\mathrm{MJ} \mathrm{m}^{-2} \mathrm{~d}^{-1}\right)$; G é o fluxo de calor no solo, assumido o valor igual a zero; $\mathrm{T}$ é a temperatura média do ar diária, medida a $2 \mathrm{~m}$ de altura $\left({ }^{\circ} \mathrm{C}\right)$; $\mathrm{u}_{2}$ é a velocidade do vento, medida a $2 \mathrm{~m}$ de altura $\left(\mathrm{m} \mathrm{s}^{-1}\right) ; \Delta$ é a declividade da curva de pressão de vapor $\left(\mathrm{kPa}{ }^{\circ} \mathrm{C}^{-1}\right)$; $\mathrm{e}_{\mathrm{s}}$ é a pressão de saturação de vapor ( $\mathrm{kPa})$; $\mathrm{e}_{\mathrm{a}}$ é a pressão de vapor real ( $\mathrm{kPa}) ; \gamma$ é a constante psicrométrica $\left(\mathrm{kPa}^{\circ} \mathrm{C}^{-1}\right)$.

Os valores de ETo foram comparados para verificar o desempenho estatístico, utilizando-se o índice de concordância d que, de acordo com Sousa (2004), foi estabelecido por Willmott et al. (1985), e estabelece uma concordância entre valores observados e estimados. O cálculo de d foi feito pela equação:

$\mathrm{d}=1-\frac{\sum_{i=1}^{\mathrm{n}}\left(\mathrm{E}_{\mathrm{i}}-\mathrm{M}_{\mathrm{i}}\right)^{2}}{\sum_{\mathrm{i}=1}^{\mathrm{n}}\left(\left|\mathrm{E}_{\mathrm{i}}^{\prime}\right|+\left|\mathrm{M}_{\mathrm{i}}^{\prime}\right|\right)^{2}}$ e $\quad \mathrm{E}_{\mathrm{i}}^{\prime}=\mathrm{E}_{\mathrm{i}}-\overline{\mathrm{M}}$ e $\mathrm{M}_{\mathrm{i}}^{\prime}=\mathrm{M}_{\mathrm{i}}-\overline{\mathrm{M}}$

em que d é o índice de Willmott, que varia de 0 a 1 ; $E_{i}$ são os eventos estimados; $M_{i}$ são os eventos medidos; e $\bar{M}$ é a média dos eventos medidos.

O índice de desempenho c é dado por c = rd, em que r é o coeficiente de correlação. O critério de julgamento do desempenho dos coeficientes médios $a$ e $b$, quando aplicados à estimativa da $R_{s}$ e respectivas ETo, foi o de Camargo \& Sentelhas (1997), para uma situação ideal $\mathrm{r}=\mathrm{d}=\mathrm{c}=1$ em que, quanto pior as estimativas, mais próximo de zero será a igualdade.

\section{Resultados e Discussão}

A análise dos dados meteorológicos de radiação global e da insolação real possibilitou a determinação dos coeficientes médios $a$ e $b$ de Angström-Prescott (Tabela 1). Os valores médios mensais de $a$ variaram de 0,241 $\pm 0,04$ (outubro) a 0,345 $\pm 0,097$ (julho). Os valores médios mensais de $b$ variaram de $0,430 \pm 0,045$ (janeiro) a $0,515 \pm 0,097$ (setembro). Tiveram, portanto, diferenças de até $36 \%$ para $a$ e de até $14 \%$ para $b$, em relação aos valores sugeridos pela FAO (Allen et al., 1998) que foi de 0,25 e 0,50 , respectivamente. O maior desvio observado nos valores de $a$ e $b$ ocorreu em agosto, $\pm 0,132 \mathrm{e} \pm 0,133$, respectivamente. O menor desvio de $a$ foi constatado em janeiro $( \pm 0,037)$ enquanto o de $b$ ocorreu em março $( \pm 0,039)$. As $R_{s}$ calculadas e observadas são valores médios mensais. As $\mathrm{R}_{\mathrm{S}}$ calculadas foram obtidas a partir de dados diários de insolação real, da radiação extraterrestre do referido dia e dos coeficientes médios de $a$ e $b$ (Tabela 1).

Evidenciou-se que nos meses mais chuvosos e quentes (outubro a abril) ocorreram os menores desvios nos coeficientes $a$ e $b$, em comparação com os meses frios e secos (maio a setembro). A incidência de poeiras e queimadas na região pode ter contribuído para a maior variabilidade dos coeficientes calculados em agosto, pois provocam maior poluição atmosférica e apresentam freqüência variável ao longo dos anos.

Os coeficientes médios para os períodos anual e geral foram 11,2 e $12,8 \%$ acima de 0,25 , e 0,4 e $2 \%$ abaixo de 0,50 , valores sugeridos pela FAO (Allen et al., 1998) para $a$ e $b$, respectivamente. Aplicando-se os valores de $a$ e $b$, obtidos nos períodos anual e geral na equação de AngströmPrescott na estimativa de $\mathrm{R}_{\mathrm{s}}$, observou-se, matematicamente, que não houve diferença na comparação entre os

Tabela 1. Valores médios mensais, anuais e de todo o período (geral) das radiações solar global $\left(\mathrm{R}_{\mathrm{s}}\right)$, observadas e calculadas pelos coeficientes $a$ e $b$ da equação de AngströmPrescott, e respectivos coeficientes de determinação $\mathrm{R}^{2}$ de $a$ e $b$, obtidos no período de 1978 a 2003, em Brasília.

\begin{tabular}{lccccc}
\hline Média & $\begin{array}{c}\mathrm{R}_{\mathrm{s}} \text { observada } \\
\left(\mathrm{MJ} \mathrm{m}^{-2} \mathrm{~d}^{-1}\right)\end{array}$ & $\begin{array}{c}\mathrm{R}_{\mathrm{s}} \text { calculada } \\
\left(\mathrm{MJ} \mathrm{m}^{-2} \mathrm{~d}^{-1}\right)\end{array}$ & $a$ & $b$ & $\mathrm{R}^{2}$ \\
\hline Janeiro & $19,20 \pm 5,32$ & $19,20 \pm 5,02$ & $0,301 \pm 0,037$ & $0,430 \pm 0,045$ & 0,958 \\
Fevereiro & $19,90 \pm 5,09$ & $19,91 \pm 4,97$ & $0,293 \pm 0,044$ & $0,460 \pm 0,049$ & 0,959 \\
Março & $18,93 \pm 5,08$ & $18,98 \pm 4,76$ & $0,284 \pm 0,046$ & $0,499 \pm 0,039$ & 0,955 \\
Abril & $18,30 \pm 4,10$ & $18,49 \pm 3,69$ & $0,304 \pm 0,045$ & $0,442 \pm 0,084$ & 0,932 \\
Maio & $17,81 \pm 3,59$ & $17,89 \pm 3,10$ & $0,301 \pm 0,054$ & $0,473 \pm 0,088$ & 0,902 \\
Junho & $18,35 \pm 3,43$ & $18,36 \pm 2,56$ & $0,311 \pm 0,049$ & $0,502 \pm 0,085$ & 0,953 \\
Julho & $18,58 \pm 2,96$ & $18,61 \pm 2,12$ & $0,345 \pm 0,097$ & $0,432 \pm 0,067$ & 0,825 \\
Agosto & $20,04 \pm 3,70$ & $19,75 \pm 3,16$ & $0,317 \pm 0,132$ & $0,449 \pm 0,133$ & 0,841 \\
Setembro & $19,81 \pm 5,06$ & $19,76 \pm 4,73$ & $0,269 \pm 0,069$ & $0,515 \pm 0,097$ & 0,933 \\
Outubro & $18,71 \pm 5,51$ & $18,71 \pm 5,23$ & $0,241 \pm 0,040$ & $0,497 \pm 0,077$ & 0,940 \\
Novembro & $17,96 \pm 5,69$ & $18,04 \pm 5,02$ & $0,264 \pm 0,062$ & $0,464 \pm 0,109$ & 0,908 \\
Dezembro & $18,40 \pm 5,13$ & $17,29 \pm 4,66$ & $0,296 \pm 0,047$ & $0,452 \pm 0,090$ & 0,918 \\
\hline Anual & $18,84 \pm 4,38$ & $18,92 \pm 4,56$ & $0,278 \pm 0,034$ & $0,498 \pm 0,052$ & 0,928 \\
\hline Geral & $18,83 \pm 4,69$ & $18,92 \pm 4,49$ & 0,282 & 0,490 & 0,810 \\
\hline
\end{tabular}

${ }^{(1)}$ Valores médios medidos. ${ }^{(2)}$ Valores estimados a partir dos respectivos coeficientes médios de $a$ e $b$. 
valores estimados e observados, o que sugere que ambos podem ser utilizados na estimativa de $\mathrm{R}_{\mathrm{s}}$.

Comparando-se os valores dos coeficientes médios anuais da equação de Angström-Prescott, determinados neste trabalho ( $a=0,278$ e $b=0,498)$, com os valores médios anuais dos Municípios de Salvador e de Viçosa (Vianello \& Alves, 1991), observa-se que, neste caso, o valor de $a$ situou-se entre os daquelas localidades. Entretanto, o valor de $b$ ficou acima e mais próximo do valor recomendado pela FAO $(0,50)$. No entanto, esta é a primeira determinação, para Brasília, dos valores desses coeficientes, daí a impossibilidade de se fazer comparações com outros resultados.

Na Figura 1, está descrita a análise de regressão entre as razões $R_{s} / R_{a}$ e $n / N$, realizada para a obtenção dos valores médios de $a$ e $b$, considerando-se o agrupamento das variáveis meteorológicas em todo o período estudado. $\mathrm{O}$ valor de $\mathrm{R}^{2}$, igual a 0,81 , indica boa relação de dependência da razão $R_{s} / R_{a}$ com a razão de insolação $\mathrm{n} / \mathrm{N}$.

Comparando-se as $\mathrm{R}_{\mathrm{S}}$ observadas, com as simuladas (Tabela 2), foi constatado, de maneira geral, que de maio a agosto o desempenho dos coeficientes médios mensais $a$ e $b$, na estimativa desta radiação, foram inferiores aos desempenhos dos outros períodos. Verifica-se que o pior desempenho na estimativa de $\mathrm{R}_{\mathrm{s}}$ ocorreu em julho, classificado como sofrível, independentemente da maneira como se obtiveram os valores de $a$ e $b$ (geral, anual, mensal e os propostos pela FAO). Os piores desempenhos verificados, nos meses de frio e de seca, podem ser indicativo de que a equação de AngströmPrescott simula melhor as $\mathrm{R}_{\mathrm{s}}$ em períodos de maior nebulosidade e umidade relativa do ar.
Nos meses de janeiro, fevereiro, março e outubro ocorreram os melhores desempenhos na estimativa da $\mathrm{R}_{\mathrm{s}}$, classificados como ótimos. A exceção foi em março, quando se usaram os coeficientes $a$ e $b$ sugeridos pela FAO, que apresentaram desempenho muito bom. Pela semelhança de desempenho no cálculo da $\mathrm{R}_{\mathrm{s}}$, independentemente de como foram obtidos os coeficientes $a$ e $b$, sugere-se o uso dos valores médios gerais para se estimar $\mathrm{R}_{\mathrm{s}}$.

Na Figura 2, estão descritos os resultados da análise dos dados da $R_{\mathrm{s}}$ observada e estimada com os coeficientes médios para os períodos anuais, geral, e o da FAO.



Figura 1. Coeficientes $a$ e $b$ que compreendem o período geral (1978 a 2003), a partir dos dados diários do quociente entre a radiação solar global e a extraterrestre $\left(\mathrm{R}_{\mathrm{s}} / \mathrm{R}_{\mathrm{a}}\right)$, decorrente da razão de insolação (n/N) e o seu respectivo $\mathrm{R}^{2}$.

Tabela 2. Desempenho dos coeficientes médios $a$ e $b$ mensal, anual e geral calculados, e dos sugeridos pela FAO, na estimativa da radiação solar global $\left(\mathrm{R}_{\mathrm{s}}\right)$, utilizando-se a equação de Angström-Prescott, com seus respectivos $\mathrm{R}^{2}$ e índices de desempenho c.

\begin{tabular}{|c|c|c|c|c|c|c|c|c|c|c|c|c|}
\hline \multirow[t]{2}{*}{ Mês } & \multicolumn{3}{|c|}{$\begin{array}{c}\text { Mensal } \\
a \text { e } b \text { calculados }^{(1)}\end{array}$} & \multicolumn{3}{|c|}{$\begin{array}{c}\text { Anual } \\
a=0,278 \text { e } b=0,498\end{array}$} & \multicolumn{3}{|c|}{$\begin{array}{c}\text { Geral } \\
a=0,282 \text { e } b=0,490\end{array}$} & \multicolumn{3}{|c|}{$\begin{array}{c}\text { FAO } \\
a=0,25 \text { e } b=0,50\end{array}$} \\
\hline & $\mathrm{R}^{2}$ & $\mathrm{c}$ & $\mathrm{D}^{(2)}$ & $\mathrm{R}^{2}$ & $\mathrm{c}$ & D & $\mathrm{R}^{2}$ & $\mathrm{c}$ & $\mathrm{D}$ & $\mathrm{R}^{2}$ & $\mathrm{c}$ & $\mathrm{D}$ \\
\hline Janeiro & 0,892 & 0,917 & OT & 0,892 & 0,916 & OT & 0,892 & 0,916 & OT & 0,892 & 0,909 & OT \\
\hline Fevereiro & 0,839 & 0,876 & OT & 0,840 & 0,876 & OT & 0,840 & 0,876 & OT & 0,840 & 0,869 & OT \\
\hline Março & 0,820 & 0,860 & OT & 0,820 & 0,860 & OT & 0,819 & 0,859 & OT & 0,821 & 0,848 & MB \\
\hline Abril & 0,636 & 0,708 & B & 0,721 & 0,779 & MB & 0,720 & 0,779 & MB & 0,723 & 0,779 & MB \\
\hline Maio & 0,635 & 0,704 & B & 0,638 & 0,709 & B & 0,637 & 0,708 & B & 0,640 & 0,700 & B \\
\hline Junho & 0,627 & 0,685 & B & 0,627 & 0,667 & B & 0,627 & 0,663 & B & 0,627 & 0,638 & MD \\
\hline Julho & 0,499 & 0,569 & $\mathrm{SF}$ & 0,504 & 0,584 & SF & 0,504 & 0,582 & $\mathrm{SF}$ & 0,506 & 0,567 & SF \\
\hline Agosto & 0,593 & 0,668 & B & 0,598 & 0,675 & B & 0,597 & 0,674 & B & 0,600 & 0,662 & B \\
\hline Setembro & 0,775 & 0,824 & $\mathrm{MB}$ & 0,775 & 0,823 & MB & 0,774 & 0,822 & MB & 0,776 & 0,815 & $\mathrm{MB}$ \\
\hline Outubro & 0,839 & 0,876 & OT & 0,839 & 0,860 & OT & 0,839 & 0,859 & OT & 0,839 & 0,875 & OT \\
\hline Novembro & 0,768 & 0,817 & $\mathrm{MB}$ & 0,768 & 0,811 & MB & 0,768 & 0,809 & $\mathrm{MB}$ & 0,768 & 0,820 & $\mathrm{MB}$ \\
\hline Dezembro & 0,813 & 0,843 & $\mathrm{MB}$ & 0,813 & 0,898 & OT & 0,813 & 0,898 & OT & 0,813 & 0,897 & OT \\
\hline
\end{tabular}

${ }^{(1)}$ Cálculo feito com os valores médios mensais de $a$ e $b$ extraídos da Tabela 1. (2)Desempenho (D): ótimo (OT), muito bom (MB), bom (B), mediano (MD) e sofrível (SF). 

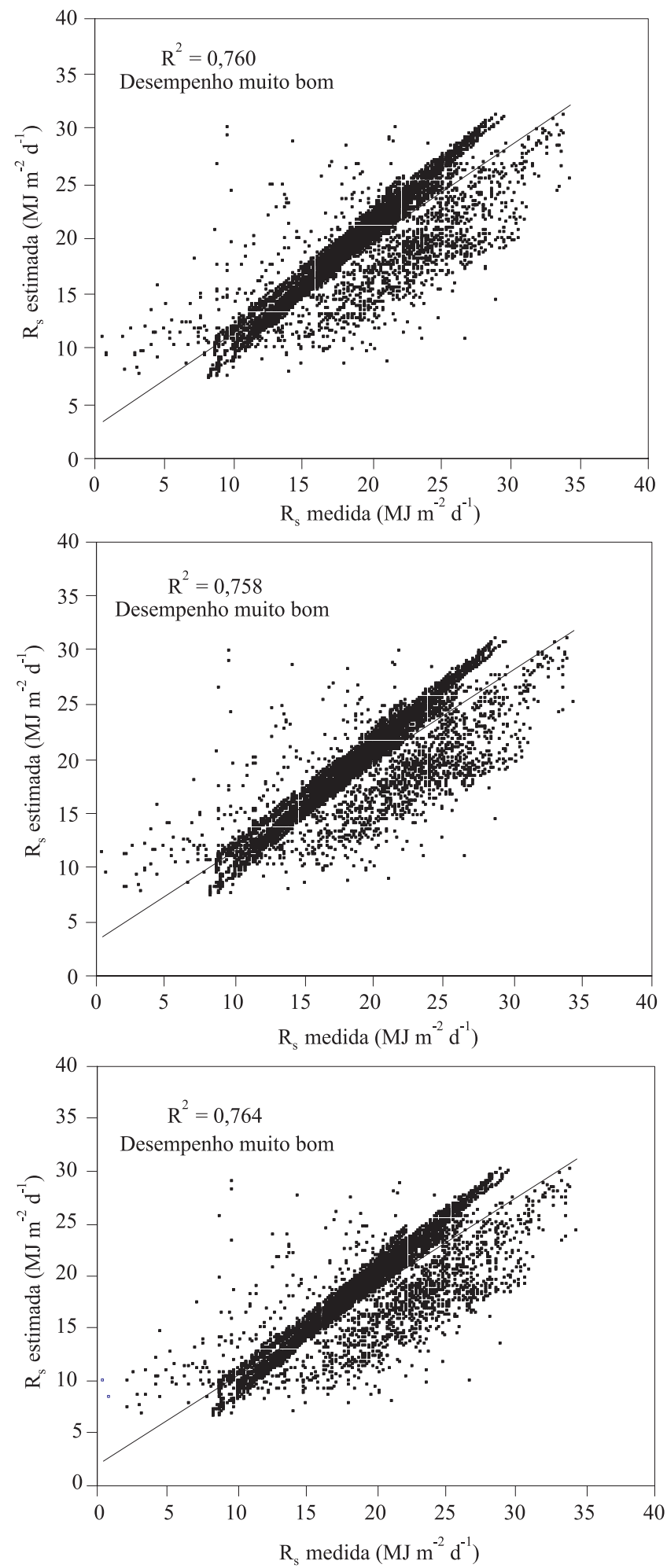

Figura 2. Comparação entre as correlações das $\mathrm{R}_{\mathrm{s}}$ medidas $\mathrm{e}$ estimadas pelos coeficientes médios anuais, geral e os sugeridos pela $\mathrm{FAO}$, com seus respectivos $\mathrm{R}^{2}$ e desempenhos.
Nela, estão apresentados dados diários dos 25 anos utilizados neste trabalho. Na análise desses dados, observa-se que o desempenho foi classificado como muito bom para o cálculo de $\mathrm{R}_{\mathrm{s}}$, embora à primeira vista, tende-se subestimar os valores simulados.

A Figura 3 exemplifica, para o mês de julho, o pior desempenho na estimativa da $\mathrm{R}_{\mathrm{s}}$, em comparação com a $R_{\mathrm{s}}$ medida; este fato é um reforço do que é observado na Tabela 2, já que esse mês é o mais frio e o de menor nebulosidade na região (Alencar, 2003).

Na Tabela 3, está descrito o desempenho do uso de $\mathrm{R}_{\mathrm{s}}$, oriundo dos diversos coeficientes $a$ e $b$ testados no cálculo da ETo. Pode-se observar que, para esta finalidade, independentemente de como se obtiveram esses coeficientes, o desempenho foi classificado como ótimo no cálculo da ETo. Entretanto, foram observados, sistematicamente, valores menores de $\mathrm{R}^{2}$ em abril e agosto, e maiores valores em janeiro, correspondentes aos períodos de início da época fria, de seca plena e pleno período chuvoso, respectivamente.

Na Figura 4 observa-se também um desempenho ótimo no cálculo da ETo, apesar de ter tido $\mathrm{R}^{2}$ um pouco menor do que aqueles da Tabela 3. A ausência de diferença de desempenho no cálculo de ETo, independentemente dos coeficientes $a$ e $b$ usados no cálculo de $\mathrm{R}_{\mathrm{s}}$, deve-se ao fato de o método de Penman-Monteith-FAO

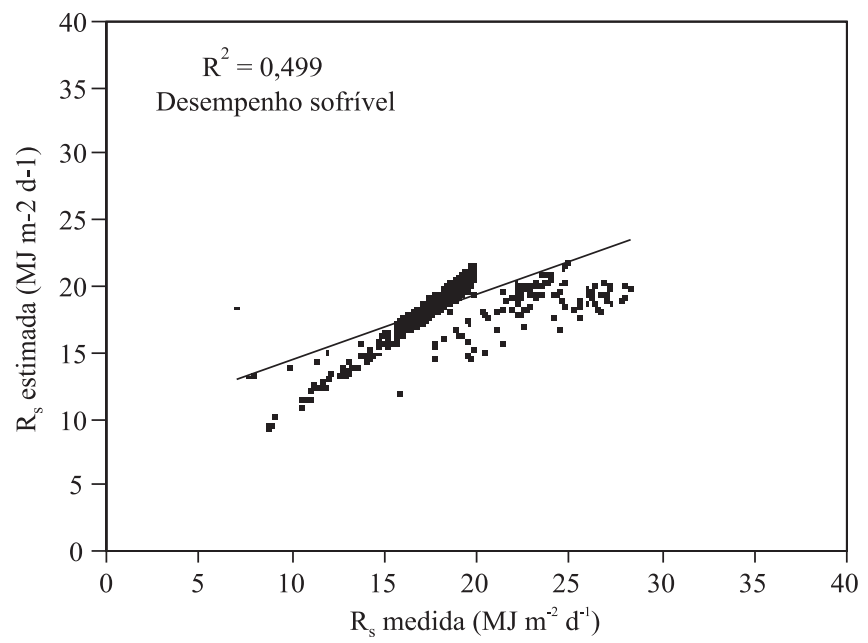

Figura 3. Comparação entre as correlações das $\mathrm{R}_{\mathrm{s}}$ medidas e estimadas pelo coeficiente médio mensal referente a julho, com seu respectivo $\mathrm{R}^{2}$ e desempenho. 
Tabela 3. Desempenho dos coeficientes médios $a$ e $b$ mensal, anual e geral, e dos sugeridos pela FAO, na estimativa da

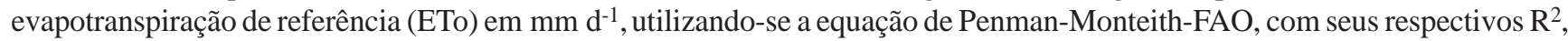
índices c e desempenho.

\begin{tabular}{|c|c|c|c|c|c|c|c|c|c|c|c|c|c|c|c|c|}
\hline \multirow[t]{2}{*}{ Mês } & \multicolumn{4}{|c|}{$\begin{array}{c}\text { Mensal } \\
a \text { e } b \text { calculados }\end{array}$} & \multicolumn{4}{|c|}{$\begin{array}{c}\text { Anual } \\
a=0,278 \text { e } b=0,498\end{array}$} & \multicolumn{4}{|c|}{$\begin{array}{c}\text { Geral } \\
a=0,282 \text { e } b=0,490\end{array}$} & \multicolumn{4}{|c|}{$\begin{array}{c}\mathrm{FAO} \\
a=0,25 \mathrm{e} b=0,50\end{array}$} \\
\hline & $\mathrm{R}^{2}$ & $\mathrm{c}$ & ETo & $\mathrm{D}^{(2)}$ & $\mathrm{R}^{2}$ & $\mathrm{c}$ & ETo & $\mathrm{D}$ & $\mathrm{R}^{2}$ & $\mathrm{c}$ & ETo & $\mathrm{D}$ & $\mathrm{R}^{2}$ & $\mathrm{c}$ & ETo & $\mathrm{D}$ \\
\hline Janeiro & 0,940 & 0,942 & 4,2 & OT & 0,942 & 0,961 & 4,2 & OT & 0,943 & 0,962 & 4,2 & OT & 0,943 & 0,959 & 4,0 & OT \\
\hline Jevereiro & 0,925 & 0,950 & 4,3 & OT & 0,923 & 0,949 & 4,3 & OT & 0,924 & 0,949 & 4,3 & OT & 0,924 & 0,947 & 4,1 & OT \\
\hline Março & 0,906 & 0,928 & 4,0 & OT & 0,906 & 0,928 & 4,0 & OT & 0,906 & 0,928 & 4,0 & OT & 0,907 & 0,923 & 3,9 & OT \\
\hline Abril & 0,865 & 0,896 & 3,9 & OT & 0,864 & 0,895 & 3,9 & OT & 0,864 & 0,895 & 3,9 & OT & 0,866 & 0,895 & 3,8 & OT \\
\hline Maio & 0,898 & 0,922 & 3,7 & OT & 0,897 & 0,921 & 3,6 & OT & 0,898 & 0,922 & 3,6 & OT & 0,899 & 0,920 & 3,6 & OT \\
\hline Junho & 0,905 & 0,925 & 3,7 & OT & 0,905 & 0,923 & 3,6 & OT & 0,905 & 0,923 & 3,6 & OT & 0,904 & 0,918 & 3,5 & OT \\
\hline Julho & 0,882 & 0,913 & 4,0 & OT & 0,905 & 0,931 & 4,0 & OT & 0,905 & 0,931 & 4,0 & OT & 0,905 & 0,930 & 3,9 & OT \\
\hline Agosto & 0,865 & 0,896 & 4,8 & OT & 0,864 & 0,895 & 4,8 & OT & 0,864 & 0,896 & 4,8 & OT & 0,864 & 0,895 & 4,7 & OT \\
\hline Setembro & 0,885 & 0,916 & 5,1 & OT & 0,885 & 0,916 & 5,1 & OT & 0,885 & 0,916 & 5,1 & OT & 0,886 & 0,917 & 5,0 & OT \\
\hline Outubro & 0,901 & 0,932 & 4,7 & OT & 0,911 & 0,935 & 4,8 & OT & 0,911 & 0,935 & 4,8 & OT & 0,910 & 0,938 & 4,7 & OT \\
\hline Novembro & 0,931 & 0,952 & 4,1 & OT & 0,929 & 0,949 & 4,3 & OT & 0,929 & 0,949 & 4,3 & OT & 0,930 & 0,952 & 4,1 & OT \\
\hline Dezembro & 0,930 & 0,953 & 4,0 & OT & 0,928 & 0,952 & 4,0 & OT & 0,928 & 0,952 & 4,0 & OT & 0,928 & 0,947 & 3,9 & OT \\
\hline
\end{tabular}

${ }^{(1)}$ Cálculo feito com os valores médios mensais de $a$ e $b$ extraídos da Tabela 1. (2)Desempenho (D): ótimo (OT).

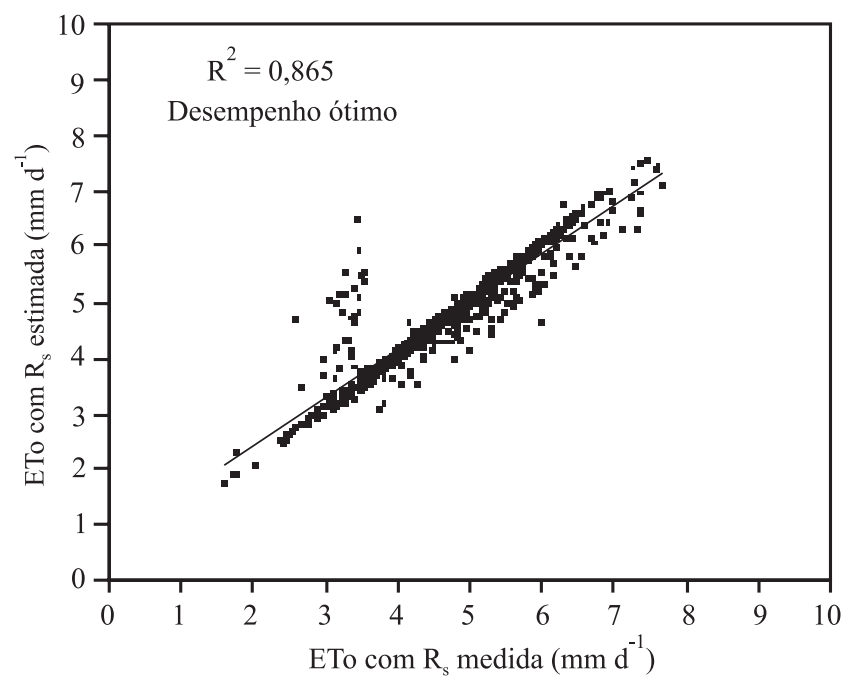

Figura 4. Comparação entre as correlações da estimativa da ETo calculada com a $\mathrm{R}_{\mathrm{s}}$ medida e a ETo calculada com as $\mathrm{R}_{\mathrm{s}}$, estimadas pelo coeficiente médio mensal, referente a agosto, seu respectivo $\mathrm{R}^{2}$ e desempenho.

ser um método combinado, que considera, além do saldo de radiação, o componente aerodinâmico do ar no processo de evapotranspiração, diminuindo, assim, a importância relativa da $\mathrm{R}_{\mathrm{s}}$.

\section{Conclusões}

1. As estimativas da radiação solar global pela equação de Angström-Prescott, com os coeficientes médios mensais $a$ e $b$, quando comparadas com os valores observados, apresentam os piores desempenhos nos meses mais frios e secos.

2. O valor de $a$ e $b$, encontrado no período geral, pode ser indicado como melhor alternativa para o cálculo da radiação solar global, em Brasília, pela equação de Angström-Prescott.

3. Não há diferenças de desempenho no cálculo da evapotranspiração de referência, quando se usa a radiação solar global calculada com os valores médios de $a$ e $b$, nos períodos mensais, anuais e geral, e os sugeridos pela FAO e a radiação solar global medida.

\section{Agradecimentos}

À Embrapa Hortaliças e ao pesquisador Neville Vianna B. dos Reis, pela cessão dos dados meteorológicos para a realização deste trabalho.

\section{Referências}

ALENCAR, D.B.S. de. Influência do total precipitado, umidade antecedente e intensidade de precipitação no escoamento superficial de uma microbacia hidrográfica do Distrito Federal. 2003. 56p. Dissertação (Mestrado) - Universidade de Brasília, Brasília.

ALLEN, R.G.; PEREIRA, L.S.; RAES, D.; SMITH, M. Crop evapotranspiration-guidelines for computing crop water requirements. Rome: FAO, 1998. 297p. (Irrigation and drainage paper, 56).

CAMARGO, A.P.; SENTELHAS, P.C. Avaliação do desempenho de diferentes métodos de estimativa da evapotranspiração potencial no Estado de São Paulo. Revista Brasileira de Agrometeorologia, v.5, p.89-97, 1997.

CRITCHFIELD, H.J. General Climatology. Englewood Cliffs: Prentice-Hall, 1974. 447p.

FONTANA, D.C.; OLIVEIRA, D. Relação entre radiação solar global e insolação para o Estado do Rio Grande do Sul. Revista Brasileira de Agrometeorologia, v.4, p.87-91, 1996. 
OMETTO, J.C.; PERRIES, A.; ITIES, B. Sur l'estimation de la radiation solaire globale et radiation nette. Versailles: Centre National de Recherche Agronomique, 1973. 11p.

PEREIRA, A.R.; ANGELOCCI, L.R.; SENTELHAS, P.C.

Agrometeorologia: fundamentos e aplicações práticas. Guaíba: Agropecuária, 2002. 478p.

SOUSA, E.T. de. Análise de dois modelos usados na simulação da infiltração e do escoamento de água em uma bacia hidrográfica do Distrito Federal. 2004. 65p. Dissertação (Mestrado) - Universidade de Brasília, Brasília.

VIANELLO, R.L.; ALVES, A.R. Meteorologia básica e aplicações. Viçosa: UFV, 1991. 449p.

WILLMOTT, C.J.; CKLESON, S.G.; DAVIS, R.E.; FEDDEMA, J.J.; KLINK, K.M.; LEGATES, D.R.; O’DONNELL, J.; ROWE, C.M. Statistics for the evaluation and comparison of models. Journal of Geophysical Research, v.90, p.8995-9005, 1985.

Recebido em 24 de março de 2005 e aprovado em 22 de março de 2006 